\title{
Topology, Crystallized
}

(Experiments): P. Dziawa et al., arXiv:1206.1705; S.-Y. Xu et al., arXiv:1206.2088 (Theory): L. Fu, PRL 106, 106802 (2011);

T. H. Hsieh et al., Nat. Comm. 3, 982 (2012).

\section{Recommended with a Commentary by Joel E. Moore, UC Berkeley and LBNL}

A key difference between quantum Hall phases induced by a magnetic field and topological insulator phases induced by spin-orbit coupling is that the latter depend crucially on a symmetry, time reversal. The action of time-reversal symmetry on electrons leads to a new kind of topological invariant in twodimensional systems [1] that takes only two possible values: if this " $\mathbb{Z}_{2}$ invariant" is even, the system is an ordinary insulator and generically has no edge state, while if it is odd, the system has a protected conducting edge state. Breaking time-reversal symmetry allows these two phases to be connected adiabatically (i.e., without closing the energy gap). The interplay of symmetry and topology is more complicated in three dimensions, particularly when crystalline point or space group symmetries are considered, and a large number of phases have been conjectured theoretically. Recent experimental photemission work by two groups confirms the existence in $\mathrm{Pb}_{1-x} \mathrm{Sn}_{x} \mathrm{Se}[2]$ and $\mathrm{Pb}_{1-x} \mathrm{Sn}_{x}$ Te [3] of one such phase, the "topological crystalline insulator"; this term was introduced by Liang Fu in a 2011 PRL [4].

In order to explain the importance of this discovery, it seems appropriate to review some prior developments in the field of three-dimensional topological invariants. With time-reversal symmetry alone, there are 4 invariants of $\mathbb{Z}_{2}$ type $[5,6,7]$, of which only one is fully stable to time-reversal-symmetric disorder. The "strong topological insulator" phase described by this invariant has a surface state, and for a perfectly crystalline surface there are an odd number of "Dirac points" enclosed by the Fermi surface, as now observed in a variety of materials [8]. There is a remarkable generalization of these results $[9,10]$ to arbitrary dimensions and to the ten symmetry classes that result from considering only time-reversal and chiral symmetry operations (the latter appear in the Bogoliubov-de Gennes description of superconductors). This generalization provides a "no-go theorem" in a sense: without including extra symmetries such as crystalline symmetries, which are not expected to be stable to disorder, the only topological phases in any dimension are in five of the ten symmetry classes, of which three are of integer type and two of $\mathbb{Z}_{2}$ type ${ }^{1}$

The question of what topological invariants exist when additional point or space group symmetries, possibly magnetic, are imposed, and what their physi-

\footnotetext{
${ }^{1}$ Strictly speaking, this is true in the arbitrary-dimension limit described by $\mathrm{K}$ theory [10]; there are additional invariants for a fixed finite number of bands, but these are not expected to be stable to disorder.
} 
cal consequences are, remains an active subject for theory with too many examples to list. The work of L. Fu mentioned above showed that when time-reversal is present along with a four-fold or six-fold rotational symmetry, there can be a distinctive electronic structure at a clean surface that respects the rotational symmetry. Rather than an odd number of surface "Dirac cones" as in topological insulators, there are quadratic surface bands that touch at a point, reminiscent of the electronic structure of bilayer graphene. The bands cannot be separated by any perturbation that respects the rotational and time-reversal symmetries. While the rotational symmetry is not stable to structural disorder, unlike timereversal, many materials can be made in sufficiently good crystalline form that this rotational symmetry is present to very high accuracy. Furthermore, it is known in other cases that there can be interesting consequences of a topological invariant when an isolated defect breaks the symmetry required for its definition.

A follow-up paper [?] proposed SnTe and similar materials as a slightly different type of topological crystalline insulator with an even number (four) of Dirac branches at the surface, resulting from band inversion at an even number of points. Mirror symmetries in the structure protects the surface states at surfaces that preserve a mirror operation. The preprints that experimentally identify a topological crystalline insulator $[2,3]$ required a number of significant steps beyond the theoretical proposal: identification and synthesis of a specific material, creation of a suitable surface for photoemission measurement, and success in identifying the characteristic state under conditions when the previously understood topological insulator phase could not provide the explanation. The experimental reason for going to doped materials, $\mathrm{Pb}_{1-x} \mathrm{Sn}_{x}$ Se with $x \approx 0.23$ in [2] and $\mathrm{Pb}_{1-x} \mathrm{Sn}_{x}$ Te with $x \approx 0.35$ in [3], is that crystals of the nominally undoped materials are in practice heavily $p$-doped from Sn vacancies; hence the surface states are unoccupied and invisible in conventional photoemission. In the doped materials, the predicted Dirac cones are clearly seen.

These experiments give hope that the theoretical effort going into "redoing crystallography with an injection of topology" may lead to other insulating and metallic phases. It would be nice to realize the first electronic structure discussed by $\mathrm{Fu}$; in general terms, topological insulators provide a surface electronic structure that is superficially similar to that of graphene but with some important differences, and a topological crystalline insulator with quadratic touching is related in the same sense to a graphene bilayer. Graphene bilayers appear to have interesting correlation physics and symmetry breaking not present in single-layer graphene, and one might ask if there are any equivalents in a thin film of a topological crystalline insulator of this type. Whatever the future holds, the present experiments add one more interesting example of the consequences of topology for electronic behavior in solids.

\section{References}

[1] C. L. Kane and E. J. Mele. $Z_{2}$ topological order and the quantum spin Hall effect. Phys. Rev. Lett., 95:146802, 2005. 
[2] P. Dziawa, B. J. Kowalski, K. Dybko, R. Buczko, A. Szczerbakow, M. Szot, E. Lusakowska, T. Balasubramanian, B. M. Wojek, M. H. Berntsen, O. Tjernberg, and T. Story. Topological crystalline insulator states in pb(1-x)sn(x)se. arXiv:1206.1705, 2012.

[3] S.-Y. Xu, Chang Liu, N. Alidoust, D. Qian, M. Neupane, J. D. Denlinger, Y. J. Wang, L. A. Wray, R. J. Cava, H. Lin, A. Marcinkova, E. Morosan, A. Bansil, and M. Z. Hasan. Observation of topological crystalline insulator phase in the lead tin chalcogenide pb1-xsnxte material class. arXiv:1206.1705, 2012.

[4] L. Fu. Topological crystalline insulators. Phys. Rev. Lett., 106:106802, 2011.

[5] J. E. Moore and L. Balents. Topological invariants of time-reversalinvariant band structures. Phys. Rev. B, 75:121306(R), 2007.

[6] L. Fu, C. L. Kane, and E. J. Mele. Topological insulators in three dimensions. Phys. Rev. Lett., 98:106803, 2007.

[7] R. Roy. Topological phases and the quantum spin hall effect in three dimensions. Phys. Rev. B, 79:195322, 2009.

[8] M. Z. Hasan and J. E. Moore. Three-dimensional topological insulators. Annual Review of Condensed Matter Physics, 2:55, 2011.

[9] A. Schnyder, S. Ryu, A. Furusaki, and A. W. W. Ludwig. Classification of topological insulators and superconductors in three spatial dimensions. Phys. Rev. B, 78:195125, 2008.

[10] A. Kitaev. Periodic table for topological insulators and superconductors. arXiv:0901.0686, 2009. 